\title{
Image formation by incoherent and coherent transition radiation from flat and rough surfaces
}

\author{
G. Stupakov \\ SLAC National Accelerator Laboratory, Stanford University, Stanford, CA \\ 94309
}

November 18, 2011

\section{INTRODUCTION}

In this paper we derive equations for the image formation of transverse profile of a relativistic beam obtained by means of optical transition radiation (OTR) from flat and rough metal surfaces. The motivation behind this study lies in the desire to suppress coherent transition radiation (COTR) observed in experiments at modern free electron lasers [1].

The physical mechanism behind the problem of COTR is that the OTR is predominantly radiated at small angles of order of $1 / \gamma$ where $\gamma$ is the relativistic factor of the beam. This means that the transverse formation size of the image is of order of $\lambda \gamma$ where $\lambda=\lambda / 2 \pi$ with $\lambda$ the radiation wavelength. For relativistic beams this can be comparable or even exceed the transverse size of the beam, which would mean that the image of the beam has very little to do with its transverse profile. It is fortuitous, however, that the incoherent image is formed by adding radiation energy of electrons and results in the transverse formation size being of order of $\lambda / \theta_{a}$, with $\theta_{a}$ is the aperture angle of the optical system [2]. The COTR image, in contrast, is formed by adding electromagnetic field of electrons, and leads to the formation size $\lambda \gamma$. In situations when the COTR intensity exceeds that of OTR the COTR imaging makes the diagnostic incapable of measuring the beam profile. 
It is clear from the previous explanation that a way to suppress COTR would be either to get rid of the central part of the radiation withing the cone $1 / \gamma$ or somehow spread it out in a wider range of angles. The former can be implemented by masking angles near the axis in the optical system [3], and the latter may be achieved by using a rough surface with local angles randomly varying from point to point by amount larger than $1 / \gamma$. Analysis of the image formation for these two cases is the subject of this paper.

\section{EQUATIONS FOR ELECTRIC FIELD OF RADIATION}

For simplicity of analysis we assume that the beam hits the surface at right angle and study the backward propagating OTR. To calculate the radiation field in the far zone we use the Kirchhoff diffraction integral [4-6] with the following equation for the electric field $\boldsymbol{E}$ radiated in the direction of vector $\boldsymbol{k}$,

$$
\boldsymbol{E}=\frac{e^{i k R}}{R} \frac{i}{2 \pi} \boldsymbol{k} \times \int e^{-i \boldsymbol{k} \cdot \boldsymbol{r}} \boldsymbol{n} \times \mathcal{E}^{\mathrm{s}}(\boldsymbol{r}) d S,
$$

where $\mathcal{E}^{\mathrm{s}}$ is the electric field generated by the currents on the surface (needed to compensate the tangential component of the incident field of the beam), $\boldsymbol{r}=(x, y)$ is the two-dimensional vector in the plane of the metal surface, $\boldsymbol{k}$ is the wave vector in the direction of the radiation, $k=|\boldsymbol{k}|=\omega / c$, and $\boldsymbol{n}$ is the unit vector perpendicular to the surface of the metal directed in vacuum. In the small-angle approximation (that is when the angle $\theta$ between $\boldsymbol{k}$ and $\boldsymbol{n}$ is small) this equation can be simplified. Introducing a $2 \mathrm{D}$ vector $\boldsymbol{\kappa}=\left(k_{x}, k_{y}\right)$ and neglecting small terms in the product $\boldsymbol{k} \times \boldsymbol{n} \times \mathcal{E}^{\mathrm{s}}$ we find:

$$
\boldsymbol{E}(\boldsymbol{\kappa})=-\frac{e^{i k R}}{R} \frac{i k}{2 \pi} \int e^{-i \boldsymbol{\kappa} \cdot r} \mathcal{E}^{\mathrm{s}} d^{2} r .
$$

Note that $\kappa \approx k \theta$. 
Considering (2) as a 2D Fourier integral we can invert it to obtain

$$
\mathcal{E}^{\mathrm{s}}=-\frac{R}{2 \pi i k} e^{-i k R} \int e^{i \boldsymbol{\kappa} \cdot \boldsymbol{r}} \boldsymbol{E} d^{2} \kappa
$$

\section{TRANSITION RADIATION OF A SINGLE ELECTRON FROM FLAT SURFACE}

Consider a relativistic electron that hits the metal at the origin of the coordinate system. As is well known, the electric field $\mathcal{E}^{\mathrm{e}}$ of the incident electron at frequency $\omega=c k$ is

$$
\mathcal{E}^{\mathrm{e}}=\frac{k e}{c \pi \gamma} K_{1}\left(\frac{k r}{\gamma}\right) \boldsymbol{\nu}
$$

with $\boldsymbol{\nu}=\boldsymbol{r} / r$ and $K_{1}$ the modified Bessel function of the second kind. The

total field $\mathcal{E}^{\mathrm{e}}+\mathcal{E}^{\mathrm{s}}$ on the surface of a perfectly conducting metal is equal to zero, hence $\mathcal{E}^{\mathrm{s}}=-\mathcal{E}^{\mathrm{e}}$. It is clear that $\boldsymbol{E}$ given by (2) is directed along $\boldsymbol{\kappa}$, that is $\boldsymbol{E}=E \boldsymbol{\kappa} / \kappa$. For $E$ we obtain

$$
\begin{aligned}
E & =\frac{e^{i k R}}{R} \frac{i}{2 \pi} \frac{k^{2} e}{c \pi \gamma} \int d^{2} r K_{1}\left(\frac{k r}{\gamma}\right) e^{-i \boldsymbol{\kappa} \cdot \boldsymbol{r}} \boldsymbol{\nu} \cdot \frac{\boldsymbol{\kappa}}{\kappa} \\
& =\frac{e^{i k R}}{R} \frac{i}{2 \pi} \frac{k^{2} e}{c \pi \gamma} \int_{0}^{\infty} r d r \int_{0}^{2 \pi} d \phi K_{1}\left(\frac{k r}{\gamma}\right) e^{-i \kappa r \cos \phi} \cos \phi .
\end{aligned}
$$

The integral over $\phi$ can be taken using

$$
\int_{0}^{2 \pi} e^{-i \alpha \cos \phi} \cos \phi d \phi=-2 \pi i J_{1}(\alpha)
$$

with the result

$$
E=\frac{e^{i k R}}{R} \frac{k^{2} e}{c \pi \gamma} \int_{0}^{\infty} K_{1}\left(\frac{k r}{\gamma}\right) J_{1}(k r \theta) r d r=\frac{e^{i k R}}{R} \frac{e}{c \pi} \frac{\theta}{\theta^{2}+\gamma^{-2}}
$$

The spectral intensity of the radiation is proportional to the quantity

$$
|E|^{2}=\left(\frac{e}{c \pi R}\right)^{2} \frac{\theta^{2}}{\left(\theta^{2}+\gamma^{-2}\right)^{2}}
$$


which is a well known angular distribution of the transition radiation from a single relativistic electron. Integrated over the angle from zero to $\theta_{m} \gg 1 / \gamma$ the intensity is

$$
2 \pi \int_{0}^{\theta_{m}}|E|^{2} \theta d \theta=2 \pi\left(\frac{e}{c \pi R}\right)^{2}\left(\ln \left(\theta_{m} \gamma\right)-\frac{1}{2}\right) .
$$

From this expression we see that the integrated intensity increases (logarithmically) with $\theta_{m}$, which means that a noticeable radiation energy goes into angles much larger than $1 / \gamma$. As we will see in the next section, this is the reason behind the ability of an OTR foil to make an image of a beam with cross sectional dimensions much smaller than $\lambda \gamma$.

\section{POINT-SPREAD FUNCTION FOR A FLAT FOIL}

In this section we will calculate the point-spread function (PSF) for a flat surface of the foil. PSF is defined as intensity distribution in an image of a single electron. To simplify consideration, we accept a simple model of the image system which consists of a lens located in the far zone and focuses the light to make an image with unit magnification. Mathematically, the electric field $\mathfrak{E}$ in the image plane of the lens is defined by the following integral [1]:

$$
\mathfrak{E}(\boldsymbol{r})=A \int_{0}^{k \theta_{a}} d^{2} \kappa \boldsymbol{E}(\boldsymbol{\kappa}) e^{i \boldsymbol{\kappa} \cdot \boldsymbol{r}},
$$

where now $\boldsymbol{r}$ is a two-dimensional vector in the image plane, $\theta_{a}$ is the collection angle for the imaging apparatus, and $A$ is a constant whose exact value we will not need. We can rewrite this integral as

$$
\mathfrak{E}(\boldsymbol{r})=A\left(\int_{0}^{\infty} d^{2} \kappa \boldsymbol{E}(\boldsymbol{\kappa}) e^{i \boldsymbol{\kappa} \cdot \boldsymbol{r}}-\int_{k \theta_{a}}^{\infty} d^{2} \kappa \boldsymbol{E}(\boldsymbol{\kappa}) e^{i \boldsymbol{\kappa} \cdot \boldsymbol{r}}\right),
$$

and then use (3) in the first integral:

$$
\mathfrak{E}(\boldsymbol{r})=A\left(-\frac{i k}{R} e^{i k R} \mathcal{E}^{\mathrm{s}}-\int_{k \theta_{a}}^{\infty} d^{2} \kappa \boldsymbol{E}(\boldsymbol{\kappa}) e^{i \boldsymbol{\kappa} \cdot \boldsymbol{r}}\right) .
$$


Assuming $\theta_{a} \gg 1 / \gamma$, for the second integral we will approximate (7) by

$$
E=\frac{e^{i k R}}{R} \frac{e}{c \pi} \frac{1}{\theta}
$$

which gives

$$
\mathfrak{E}(\boldsymbol{r})=A \frac{e^{i k R}}{R}\left(\frac{2 i k^{2} e}{c \gamma} K\left(\frac{k r}{\gamma}\right) \boldsymbol{\nu}-\frac{e}{c \pi} \int_{k \theta_{a}}^{\infty} d^{2} \kappa \frac{\boldsymbol{\kappa}}{\kappa} \frac{1}{\theta} e^{i \boldsymbol{\kappa} \cdot \boldsymbol{r}}\right) .
$$

The second integral in this equation will be directed along $\boldsymbol{\nu}=\boldsymbol{r} / r$,

$$
\int_{k \theta_{a}}^{\infty} d^{2} \kappa \frac{\kappa}{\kappa} \frac{1}{\theta} e^{i \kappa \cdot \boldsymbol{r}}=B \boldsymbol{\nu}
$$

with

$$
B=\int_{k \theta_{a}}^{\infty} \kappa d \kappa \frac{1}{\theta} \int_{0}^{2 \pi} d \phi \cos \phi e^{i \kappa r \cos \phi}=2 \pi i k \frac{1}{r} J_{0}\left(k r \theta_{a}\right) .
$$

This gives for $\mathfrak{E}(\boldsymbol{r})$

$$
\mathfrak{E}(\boldsymbol{r})=\operatorname{Ai\nu } \frac{2 k e}{c} \frac{e^{i k R}}{R}\left(\frac{k}{\gamma} K_{1}\left(\frac{k r}{\gamma}\right)-\frac{1}{r} J_{0}\left(k r \theta_{a}\right)\right) .
$$

In the limit $\gamma \rightarrow \infty$ one can approximate $K_{1}(k r / \gamma) \approx \gamma / k r$ with the following result

$$
\mathfrak{E}=A i \nu \frac{2 k e}{c} \frac{e^{i k R}}{R} \frac{1-J_{0}\left(k r \theta_{a}\right)}{r}
$$

This function has a zero value at $r=0$ and decays with $r$ as $1 / r$ at large distances.

The distribution of the intensity on the image screen is proportional to

$$
I_{\mathrm{PSF}} \equiv \mathfrak{E} \cdot \mathfrak{E}^{*}=|A|^{2} \frac{4 k^{4} e^{2}}{R^{2} c^{2}} \theta_{a}^{2} \frac{\left(1-J_{0}(\xi)\right)^{2}}{\xi^{2}},
$$

with $\xi=k r \theta_{a}$. As follows from (19) the spot size is of order of $\xi \sim 1$, or $r \sim 1 / k \theta_{a}$. To find the total energy in the spot size, we need to integrate the quantity $\int_{0}^{\infty} d \xi \xi \mathfrak{E} \cdot \mathfrak{E}^{*}$. Note that with expression (19) the integral diverges 
at $\xi \rightarrow \infty$ which means that for energy calculations one should use a more accurate expression (17) for the field. Analysis shows that with that expression the energy integral converges at $r \sim \lambda \gamma$ (that is the transverse formation size), and involves $\ln \gamma$.

Our analysis can be easily extended to the case when there is a mask on the lens that blocks small angles. Let us assume that the mask blocks radiation from the foil propagating within $0<\theta<\theta_{0}$. In this case the integral (10) extends from $\theta_{0}$ to $\theta_{a}$ and Eq. (18) is replaced by

$$
\mathfrak{E}=A i \nu \frac{2 k e}{c} \frac{e^{i k R}}{R} \frac{J_{0}\left(k r \theta_{0}\right)-J_{0}\left(k r \theta_{a}\right)}{r} .
$$

This function oscillates and decays at large distances as $r^{-3 / 2}$. The integral $\int_{0}^{\infty} d \xi \xi \mathfrak{E} \cdot \mathfrak{E}^{*}$ with $\mathfrak{E}$ given by $(20)$ now converges and gives finite energy in the field of the image.

For illustration, in Fig. 1 we plot the functions $\theta_{a}^{2}\left(1-J_{0}(\xi)\right)^{2} / \xi^{2}$ (blue line) and $\left.\theta_{a}^{2}\left(J_{0}\left(\frac{1}{2} \xi\right)\right)^{2}-J_{0}(\xi)\right)^{2} / \xi^{2}$ (red line) for $1 / k=0.1 \mu \mathrm{m}$ and $\theta_{a}=100 \mathrm{mrad}$ (both functions are multiplied by $10^{3}$ ).

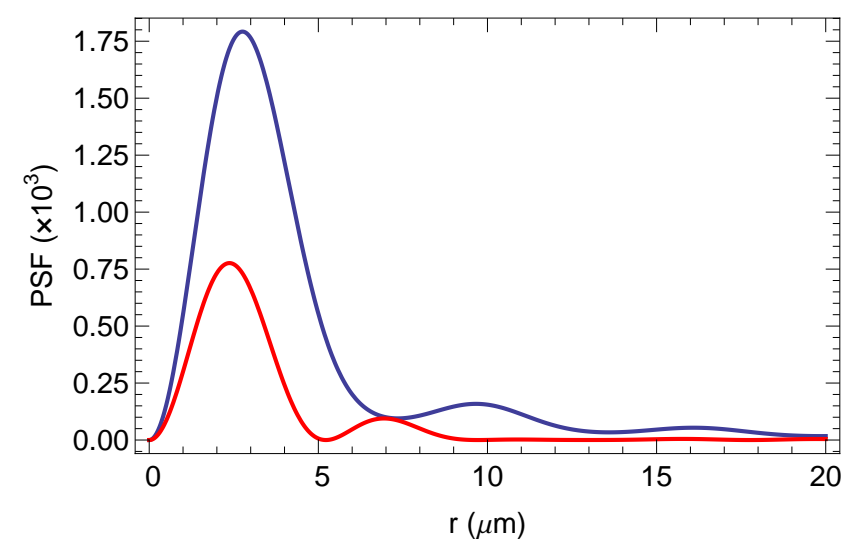

FIG. 1: PSF functions for a flat surface. 


\section{IMAGE OF TRANSVERSE BEAM PROFILE FOR A FLAT SUR- FACE}

Consider now an image generated by a beam consisting of many electrons hitting the foil. The electric field of a single electron hitting the foil at point $\boldsymbol{r}_{n}$ will result in the electric field on the detector given by (18) with the origin shifted from zero to $\boldsymbol{r}_{n}$

$$
\mathfrak{E}_{n}\left(\boldsymbol{r}, \boldsymbol{r}_{n}, z_{n}\right)=A i \frac{2 k e}{c} \frac{e^{i k R}}{R} e^{i k z_{n}} \frac{\boldsymbol{r}-\boldsymbol{r}_{n}}{\left|\boldsymbol{r}-\boldsymbol{r}_{n}\right|} \frac{1-J_{0}\left(k\left|\boldsymbol{r}-\boldsymbol{r}_{n}\right| \theta_{a}\right)}{\left|\boldsymbol{r}-\boldsymbol{r}_{n}\right|} .
$$

In this equation we also added the factor $e^{i k z_{n}}$ which is the phase due to the arrival time at the foil for an electron that has the longitudinal coordinate $z_{n}$ in the bunch.

In what follows we calculate the averaged intensity distribution of the image by averaging

$$
\sum_{n, l=1}^{N} \mathfrak{E}_{n} \cdot \mathfrak{E}_{l}^{*}=\sum_{n=1}^{N}\left|\mathfrak{E}_{n}\right|^{2}+\sum_{n \neq l}^{N} \mathfrak{E}_{n} \cdot \mathfrak{E}_{l}^{*}
$$

over the distribution function of electrons in the beam. Here $N$ is the total number of electrons. To carry out the averaging we need a two-particle distribution function $f_{2}\left(\boldsymbol{r}, z, \boldsymbol{r}^{\prime}, z^{\prime}\right)$ which gives the probability that in a pair of

electrons one is located at $\boldsymbol{r}, z$ and the other one at $\boldsymbol{r}^{\prime}, z^{\prime}$. Following a standard representation in statistical physics, we write $f_{2}$ as a product of one-particle distributions plus a correlation function $g$

$$
f_{2}\left(\boldsymbol{r}, z, \boldsymbol{r}^{\prime}, z^{\prime}\right)=f_{1}(\boldsymbol{r}, z) f_{1}\left(\boldsymbol{r}^{\prime}, z^{\prime}\right)+g\left(\boldsymbol{r}, z, \boldsymbol{r}^{\prime}, z^{\prime}\right) .
$$

Using the distribution function (23) the averaging is carried out as

$$
\begin{aligned}
& I(\boldsymbol{r})=N \int d^{2} r_{1} d z_{1} f_{1}\left(\boldsymbol{r}_{1}, z_{1}\right)\left|\mathfrak{E}_{1}\left(\boldsymbol{r}, \boldsymbol{r}_{1}, z_{1}\right)\right|^{2} \\
& +N(N-1) \int d^{2} r_{1} d z_{1} d^{2} r_{2} d z_{2} \mathfrak{E}_{1}\left(\boldsymbol{r}, \boldsymbol{r}_{1}, z_{1}\right) \cdot \mathfrak{E}_{2}\left(\boldsymbol{r}, \boldsymbol{r}_{2}, z_{2}\right)^{*} \\
& \times\left[f_{1}\left(\boldsymbol{r}_{\mathbf{1}}, z_{1}\right) f_{1}\left(\boldsymbol{r}_{2}, z_{2}\right)+g\left(\boldsymbol{r}_{1}, z_{1}, \boldsymbol{r}_{2}, z_{2}\right)\right] .
\end{aligned}
$$


Let us consider the first term in (24) which we denote by $I_{\text {incoh }}$. It is due to the incoherent transition radiation. Note that $\left|\mathfrak{E}_{1}\left(\boldsymbol{r}, \boldsymbol{r}_{1}, z_{1}\right)\right|^{2}$ does not depend on $z_{1}$. Using (18) we obtain for this term

$$
I_{\text {incoh }}(\boldsymbol{r})=N|A|^{2} \frac{4 k^{2} e^{2}}{R^{2} c^{2}} \int d^{2} r_{1} \tilde{f}_{1}\left(\boldsymbol{r}_{1}\right) \frac{\left(1-J_{0}\left(k \theta_{a}\left|\boldsymbol{r}-\boldsymbol{r}_{1}\right|\right)\right)^{2}}{\left|\boldsymbol{r}-\boldsymbol{r}_{1}\right|^{2}},
$$

where $\tilde{f}_{1}=\int d z f_{1}$. For illustration we show in Fig. 2 the plot of $I_{\text {incoh }}(r)$ for

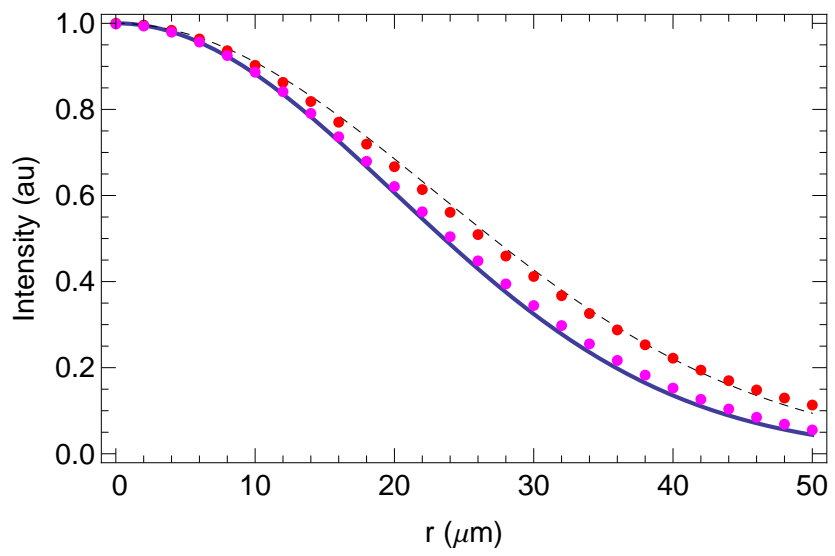

FIG. 2: Comparison of the incoherent image (red dots) with the original beam profile (blue line). We assume $\lambda / \theta_{a}=1 \mu \mathrm{m}$ and $\sigma_{x}=\sigma_{y}=20 \mu \mathrm{m}$. The dashed line shows a Gaussian fit to the image with $\sigma_{x}=\sigma_{y}=23 \mu \mathrm{m}$. Magenta dots show the image with the central part of radiation masked $\left(\theta_{0}=\frac{1}{2} \theta_{a}\right)$ and intensity multiplied by a factor of 4.6. One can see that the masked image has a better representation of the beam profile compared with the one without the mask.

a beam with a Gaussian profile with rms transverse sizes $\sigma_{x}=\sigma_{y}=20 \mu \mathrm{m}$. For the particular choice of parameters indicated in the caption to the figure, the measured profile has $\sigma_{x}=\sigma_{y}=23 \mu \mathrm{m}$ - close to the original values of the beam parameters.

Let us consider now the second term in (24), due to coherent transition radiation, which we denote by $I_{\text {coh }}$. We assume that the 1-particle distribution 
function $f_{1}$ is a smooth function of $z$ and has a negligible harmonic contents at the wavenumber $k$. Then the only contribution comes from the $g$ term in (24),

$$
I_{\mathrm{coh}}(\boldsymbol{r})=N^{2} \int d^{2} r_{1} d z_{1} d^{2} r_{2} d z_{2} g\left(\boldsymbol{r}_{1}, z_{1}, \boldsymbol{r}_{2}, z_{2}\right) \mathfrak{E}_{1}\left(\boldsymbol{r}, \boldsymbol{r}_{1}, z_{1}\right) \cdot \mathfrak{E}_{2}\left(\boldsymbol{r}, \boldsymbol{r}_{2}, z_{2}\right)^{*}
$$

where we replaced $N(N-1) \approx N^{2}$.

To simplify calculations, we will focus on a simple case when

$$
f_{2}\left(\boldsymbol{r}, z, \boldsymbol{r}^{\prime}, z^{\prime}\right)=f_{1}(\boldsymbol{r}) f_{1}\left(\boldsymbol{r}^{\prime}\right)\left(1+g\left(z-z^{\prime}\right)\right)
$$

This is the case of a beam that on average is uniform in $z$ with particles' positions correlated in that direction, while there are no correlations in the radial direction. The model (27) is reasonable when the correlation length along the bunch is much smaller than the bunch length - a typical situation for OTR with submicron wavelengths. For the model (27) we introduce

$$
\hat{g}(k)=\int d z e^{i k z} g\left(z_{1}-z_{2}\right)
$$

Using (21) in (26) we obtain

$$
I_{\mathrm{coh}}(\boldsymbol{r})=|A|^{2} \frac{4 k^{2} e^{2}}{R^{2} c^{2}} N^{2} L \hat{g}(k)\left(\int d^{2} r_{1} f_{1}\left(\boldsymbol{r}_{1}\right) \frac{\left(\boldsymbol{r}-\boldsymbol{r}_{1}\right)}{\left|\boldsymbol{r}-\boldsymbol{r}_{1}\right|^{2}}\left[1-J_{0}\left(k\left|\boldsymbol{r}-\boldsymbol{r}_{1}\right| \theta_{a}\right)\right]\right)^{2}
$$

where $L$ is the length of the bunch. The integral on the right hand side is not localized around point $\boldsymbol{r}$ - the whole beam area contributes to the light at one point of the image.

For further comparison with rough surface (considered in the following sections) it is convenient to separate the factor

$$
|A|^{2} \frac{4 k^{4} e^{2}}{R^{2} c^{2}} N^{2} L \hat{g}(k)
$$


from the integral in (29) and redefine $I_{\text {coh }}$ as dimensionless quantity

$$
I_{\mathrm{coh}}=k^{-2}\left(\int d^{2} r_{1} f_{1}\left(\boldsymbol{r}_{1}\right) \frac{\left(\boldsymbol{r}-\boldsymbol{r}_{1}\right)}{\left|\boldsymbol{r}-\boldsymbol{r}_{1}\right|^{2}}\left[1-J_{0}\left(k\left|\boldsymbol{r}-\boldsymbol{r}_{1}\right| \theta_{a}\right)\right]\right)^{2} .
$$

In case of a mask that blocks radiation at $0<\theta<\theta_{0}$, Eq. (31) is replaced by

$$
I_{\mathrm{coh}}=k^{-2}\left(\int d^{2} r_{1} f_{1}\left(\boldsymbol{r}_{1}\right) \frac{\left(\boldsymbol{r}-\boldsymbol{r}_{1}\right)}{\left|\boldsymbol{r}-\boldsymbol{r}_{1}\right|^{2}}\left[J_{0}\left(k\left|\boldsymbol{r}-\boldsymbol{r}_{1}\right| \theta_{0}\right)-J_{0}\left(k\left|\boldsymbol{r}-\boldsymbol{r}_{1}\right| \theta_{a}\right)\right]\right)^{2} .
$$

This result immediately follows from (31) if one compares (18) and (20). To analyze (32) we will note that

$$
\frac{\boldsymbol{r}}{|\boldsymbol{r}|^{2}}\left[J_{0}\left(k r \theta_{0}\right)-J_{0}\left(k r \theta_{a}\right)\right]=\frac{1}{2 \pi i} \int_{k \theta_{0}}^{k \theta_{a}} d^{2} \kappa \frac{\boldsymbol{\kappa}}{\kappa^{2}} e^{i \boldsymbol{\kappa} \cdot \boldsymbol{r}},
$$

and rewrite $(32)$ as

$$
I_{\mathrm{coh}}=\left(\frac{1}{2 \pi k}\right)^{2}\left|\int d^{2} r_{1} f_{1}\left(\boldsymbol{r}_{1}\right) \int_{k \theta_{0}}^{k \theta_{a}} d^{2} \kappa \frac{\boldsymbol{\kappa}}{\kappa^{2}} e^{i \boldsymbol{\kappa} \cdot\left(\boldsymbol{r}-\boldsymbol{r}_{1}\right)}\right|^{2} .
$$

Assuming a Gaussian beam profile

$$
f_{1}(\boldsymbol{r})=\frac{1}{2 \pi \sigma^{2}} e^{-r^{2} / 2 \sigma^{2}}
$$

and using

$$
\frac{1}{2 \pi \sigma^{2}} \int d^{2} r_{1} e^{-r_{1}^{2} / 2 \sigma^{2}} e^{-i \kappa \cdot r_{1}}=e^{-\kappa^{2} \sigma^{2} / 2}
$$

we obtain

$$
I_{\mathrm{coh}}=\left(\frac{1}{2 \pi k}\right)^{2}\left|\int_{k \theta_{0}}^{k \theta_{a}} d^{2} \kappa \frac{\boldsymbol{\kappa}}{\kappa^{2}} e^{i \kappa \cdot \boldsymbol{r}} e^{-\kappa^{2} \sigma^{2} / 2}\right|^{2}
$$

This integral becomes exponentially small when $k \sigma \theta_{0}, k \sigma \theta_{a} \gg 1$.

Let us return to the case of no mask, $\theta_{0}=0$, and assume that and $k \theta_{a} \sigma \gg 1$. In this limit we can then replace the upper limit in the integral by infinity,

$$
I_{\mathrm{coh}}=\left(\frac{1}{2 \pi k}\right)^{2}\left|\int_{0}^{\infty} d^{2} \kappa \frac{\boldsymbol{\kappa}}{\kappa^{2}} e^{i \kappa \cdot \boldsymbol{r}} e^{-\kappa^{2} \sigma^{2} / 2}\right|^{2}=\left(\frac{1}{k r}\right)^{2}\left(1-e^{-r^{2} / 2 \sigma^{2}}\right)^{2} .
$$


This function is shown in Fig. 3 by a solid blue line. It indicates a "doughnut" shape of the image with a dark region near the center and illuminated ring ${ }^{1}$.

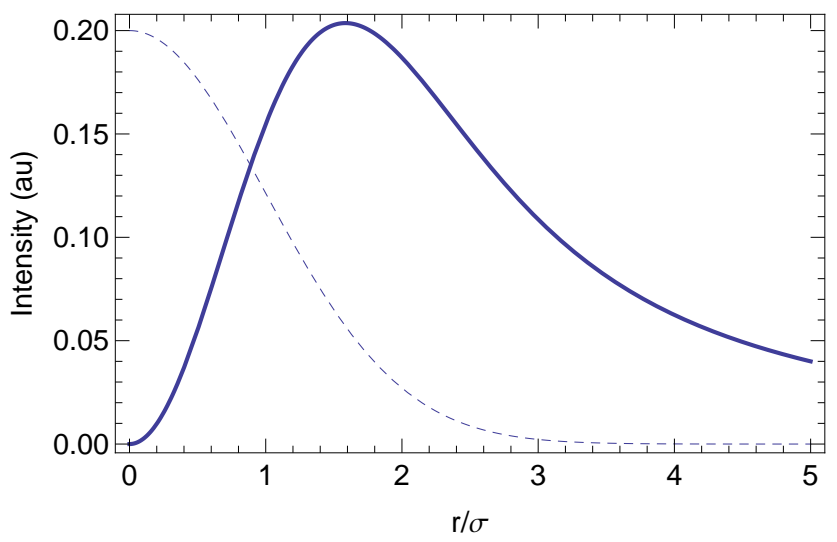

FIG. 3: Comparison of the coherent image (solid blue line) with the (Gaussian) beam profile (dashed line). Plotted is the quantity $I_{\mathrm{coh}}(k \sigma)^{2}$.

Let us also consider a flat beam profile of radius $R$

$$
f_{1}(\boldsymbol{r})=\frac{1}{\pi R^{2}} h(R-r),
$$

with $h$ the step function defined by $h(x)=1$ for $x \geq 0$ and $h(x)=0$ for $x<0$. Note that the rms size of such a beam is $\sigma_{x}=\sigma_{y}=\sigma=\frac{1}{2} R$. Using

$$
\begin{aligned}
\frac{1}{\pi R^{2}} \int d^{2} r_{1} h\left(r_{1}-R\right) e^{-i \kappa \cdot r_{1}} & =\frac{1}{\pi R^{2}} \int_{0}^{R} r_{1} d r_{1} \int_{0}^{2 \pi} d \phi e^{-i \kappa r_{1} \cos \phi} \\
& =\frac{2}{R \kappa} J_{1}(\kappa R),
\end{aligned}
$$

\footnotetext{
${ }^{1}$ The "doughnut" shape of COTR image was also derived in [1], where it was shown that $I_{\text {coh }}$ is proportional to the gradient of the transverse distribution $f_{1}$. However, this result was obtained in the limit $\sigma \gg \lambda \gamma$, while in this paper we assume an opposite inequality (that is we consider the limit $\gamma \rightarrow \infty$ ), more appropriate for highly relativistic beams.
} 
we obtain (again assuming a mask covering $\theta<\theta_{0}$ )

$$
\begin{aligned}
I_{\mathrm{coh}} & =\left(\frac{1}{2 \pi k}\right)^{2}\left|\int_{k \theta_{0}}^{k \theta_{a}} d^{2} \kappa \frac{\kappa}{\kappa^{2}} e^{i \kappa \cdot r} \frac{2}{R \kappa} J_{1}(\kappa R)\right|^{2} \\
& =4\left(\frac{1}{k R}\right)^{2}\left|\int_{k \theta_{0}}^{k \theta_{a}} d \kappa J_{1}(\kappa r) \frac{1}{\kappa} J_{1}(\kappa R)\right|^{2}
\end{aligned}
$$

The plot of this function for $\theta_{0}=\frac{1}{2} \theta_{a}$ and $R=20 / k \theta_{a}$ is shown in Fig. 4 . There is clearly no resemblance of this image to the original Gaussian profile

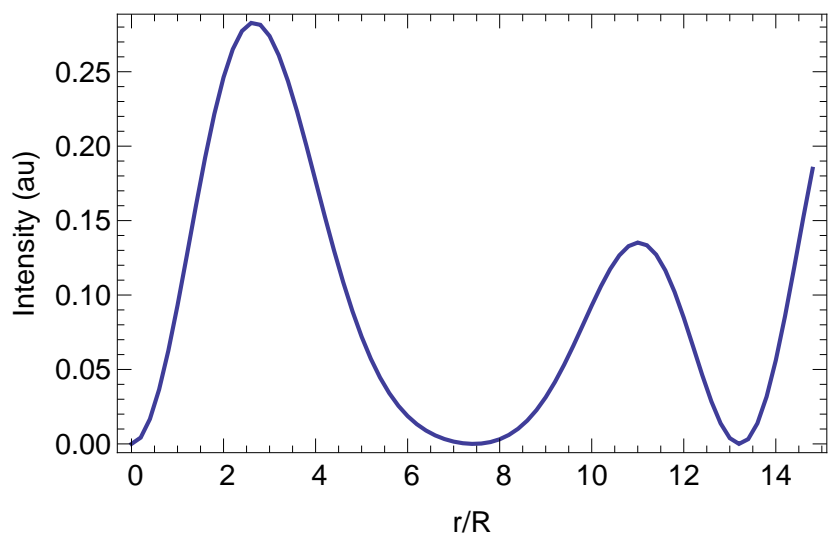

FIG. 4: Coherent image of a flat profile with a mask. Plotted is the quantity $10^{5} I_{\mathrm{coh}}(k R)^{2}$.

of the beam.

\section{DIFFRACTION RADIATION FROM A ROUGH SURFACE}

Let's consider a rough surface whose local height at point $(x, y)$ deviates from a flat one by $\xi(x, y)$ where $\xi(x, y)$ is a normally distributed random field with $\langle\xi\rangle=0$. Illustration of a random surface profile is given by Fig. 5 .

Deviation of the metal surface from a flat one modifies the Kirchhoff integral (1) in two places. First, it adds an additional phase factor $e^{-i k \xi(\boldsymbol{r})}$ under 

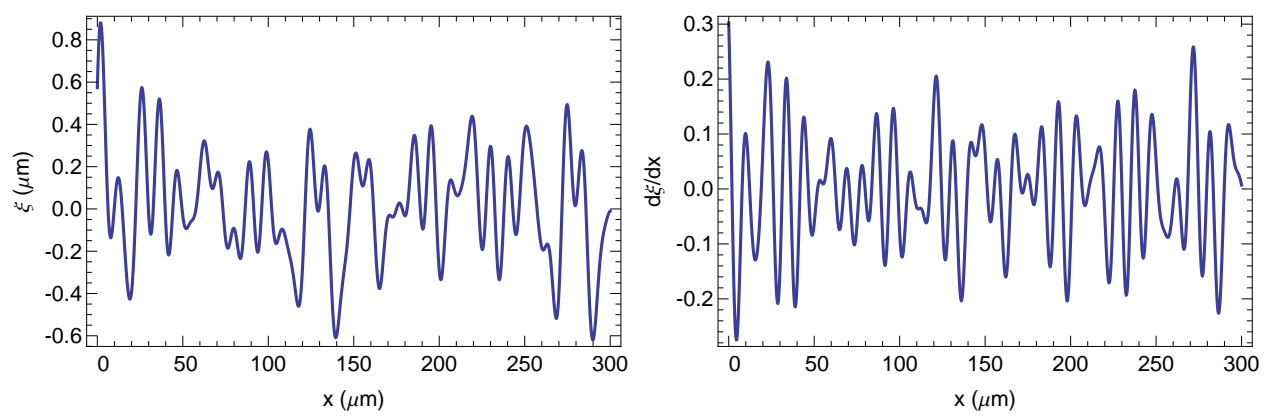

FIG. 5: Illustration of a rough surface profile: the function $\xi$ versus $x$ (left plot) and the angle $d \xi / d x$ versus $x$ (right plot).

the integral due to the increased (for $\xi<0$ ) or decreased (for $\xi>0$ ) path needed for a wave to propagate from the surface element at point $x, y$. Second, the normal vector $n$ to the surface now varies from one point to another. This second effect is small if we assume that the characteristic value of the roughness angle, $|\nabla \xi|$, is small. We will neglect it in what follows. Finally, one has to add an additional phase $e^{-i k \xi(\boldsymbol{r})}$ to the right hand side of (4) for the field of the electron at the surface of the metal. Combining both phase factors, instead of (5) we obtain

$$
\boldsymbol{E}(\boldsymbol{\kappa})=\frac{\boldsymbol{\kappa}}{\kappa} \frac{e^{i k R}}{R} \frac{i}{2 \pi} \frac{k^{2} e}{c \pi \gamma} \int d^{2} r K_{1}\left(\frac{k r}{\gamma}\right) e^{-2 i k \xi(\boldsymbol{r})} e^{-i \boldsymbol{\kappa} \cdot \boldsymbol{r}} \boldsymbol{\nu} \cdot \frac{\boldsymbol{\kappa}}{\kappa} .
$$

To calculate the spectral intensity of radiation $\boldsymbol{E} \cdot \boldsymbol{E}^{*}$ we will average it over various realizations of the roughness profiles denoting the averaging with angular brackets,

$$
\begin{aligned}
\left\langle\boldsymbol{E} \cdot \boldsymbol{E}^{*}\right\rangle & =\frac{1}{4 \pi^{2} R^{2}} \frac{k^{2} e^{2}}{\pi^{2} c^{2} \gamma^{2} \theta^{2}} \iint K_{1}\left(\frac{k r}{\gamma}\right) K_{1}\left(\frac{k r^{\prime}}{\gamma}\right) e^{-i \boldsymbol{\kappa} \cdot\left(\boldsymbol{r}-\boldsymbol{r}^{\prime}\right)} \\
& \times\left\langle e^{2 i k\left(\xi(\boldsymbol{r})-\xi\left(\boldsymbol{r}^{\prime}\right)\right)}\right\rangle(\boldsymbol{\nu} \cdot \boldsymbol{\kappa})\left(\boldsymbol{\nu}^{\prime} \cdot \boldsymbol{\kappa}\right) d^{2} r d^{2} r^{\prime}
\end{aligned}
$$

For a normally distributed roughness field with $\langle\xi(\boldsymbol{r})\rangle=0$ the following equa- 
tion holds [7],

$$
\left\langle e^{2 i k\left(\xi(\boldsymbol{r})-\xi\left(\boldsymbol{r}^{\prime}\right)\right)}\right\rangle=e^{-2 k^{2}\left\langle\left[\xi(\boldsymbol{r})-\xi\left(\boldsymbol{r}^{\prime}\right)\right]^{2}\right\rangle}=e^{-4 k^{2} \sigma_{\xi}^{2}} e^{4 k^{2} \psi\left(\left|\boldsymbol{r}-\boldsymbol{r}^{\prime}\right|\right)},
$$

where $\psi$ is the correlation function of the field, and $\sigma_{\xi}$ is the rms height of roughness. In what follows we will assume a simple exponential form for the correlation function,

$$
\psi(r)=\sigma_{\xi}^{2} e^{-\frac{r^{2}}{r_{0}^{2}}}
$$

with $r_{0}$ the correlation distance.

Let us assume that we are dealing with roughness which has the property $k \sigma_{\xi} \gg 1$. Rewriting this inequality as $\sigma_{\xi} \gg \lambda$, we see that it means that the rms roughness height is much larger than the reduced wavelength of light. This is an important condition - when it is satisfied, all the emitted light has a diffuse character. In the opposite case most of the light is specular, and the situation is not much different from a smooth flat foil. In the limit $k \sigma_{\xi} \gg 1$ the main contribution to the integral (43) comes from the region $\left|\boldsymbol{r}-\boldsymbol{r}^{\prime}\right| \ll r_{0}$ and we can expand the exponential function in $(45), \psi(r) \approx \sigma_{\xi}^{2}\left(1-r^{2} / r_{0}^{2}\right)$, and use

$$
\left\langle e^{2 i k\left(\xi(\boldsymbol{r})-\xi\left(\boldsymbol{r}^{\prime}\right)\right)}\right\rangle \approx e^{-4 k^{2} \sigma_{\xi}^{2}\left|\boldsymbol{r}-\boldsymbol{r}^{\prime}\right|^{2} / r_{0}^{2}}
$$

We will also require $1 \ll r_{0} / \sigma_{\xi} \ll \gamma$. Noting that $\theta_{r} \equiv \sigma_{\xi} / r_{0}$ has the meaning of the characteristic slope of the roughness, this condition can also be written as

$$
1 \gg \theta_{r} \gg 1 / \gamma
$$

that is the slope is larger than $1 / \gamma$, but much smaller than one. 


\section{PSF FOR A ROUGH SURFACE}

We can now calculate the PSF for a rough OTR surface. The equation for the image field is given by (10) with $\boldsymbol{E}(\boldsymbol{\kappa})$ defined by (42). Combining these two equations we find for the intensity of the image $\mathfrak{E} \cdot \mathfrak{E}^{*}$ (which is, as before, defined as $I$ ) the following expression,

$$
\begin{aligned}
I(\boldsymbol{r}) & \equiv \mathfrak{E}(\boldsymbol{r}) \cdot \mathfrak{E}^{*}(\boldsymbol{r})=\left(\frac{1}{2 \pi R} \frac{k^{2} e}{c \pi \gamma} A\right)^{2} \int_{0}^{k \theta_{a}} d^{2} \kappa e^{i \boldsymbol{\kappa} \cdot \boldsymbol{r}} \frac{\boldsymbol{\kappa}}{\kappa^{2}} \\
& \times \int d^{2} r^{\prime} K_{1}\left(\frac{k r^{\prime}}{\gamma}\right) e^{-2 i k \xi\left(\boldsymbol{r}^{\prime}\right)} e^{-i \boldsymbol{\kappa} \cdot \boldsymbol{r}^{\prime}} \boldsymbol{\nu}^{\prime} \cdot \boldsymbol{\kappa} \\
& \cdot \int_{0}^{k \theta_{a}} d^{2} \kappa^{\prime} e^{-i \boldsymbol{\kappa}^{\prime} \cdot \boldsymbol{r}} \frac{\boldsymbol{\kappa}^{\prime}}{\kappa^{\prime 2}} \int d^{2} r^{\prime \prime} K_{1}\left(\frac{k r^{\prime \prime}}{\gamma}\right) e^{-2 i k \xi\left(\boldsymbol{r}^{\prime \prime}\right)} e^{-i \boldsymbol{\kappa}^{\prime} \cdot \boldsymbol{r}^{\prime \prime}} \boldsymbol{\nu}^{\prime \prime} \cdot \boldsymbol{\kappa}^{\prime}
\end{aligned}
$$

We average $I$ over the roughness, replace $K_{1}$ by its asymptotic expression in the limit of large $\gamma, K_{1}(k r / \gamma) \approx \gamma / k r$, and use (46):

$$
\begin{aligned}
\langle I(\boldsymbol{r})\rangle & =\left(\frac{1}{2 \pi R} \frac{k e}{c \pi} A\right)^{2} \int_{0}^{k \theta_{a}} d^{2} \kappa d^{2} \kappa^{\prime} \frac{\boldsymbol{\kappa}^{\prime} \cdot \boldsymbol{\kappa}}{\kappa^{\prime 2} \kappa^{2}} e^{i\left(\boldsymbol{\kappa}-\boldsymbol{\kappa}^{\prime}\right) \cdot \boldsymbol{r}} \\
& \times \int \frac{d^{2} r^{\prime}}{r^{\prime}} \frac{d^{2} r^{\prime \prime}}{r^{\prime \prime}}\left\langle e^{-2 i k\left(\xi\left(\boldsymbol{r}^{\prime}\right)-\xi\left(\boldsymbol{r}^{\prime \prime}\right)\right)}\right\rangle e^{i \boldsymbol{\kappa}^{\prime} \cdot \boldsymbol{r}^{\prime \prime}-i \boldsymbol{\kappa} \cdot \boldsymbol{r}^{\prime}}\left(\boldsymbol{\nu}^{\prime} \cdot \boldsymbol{\kappa}\right)\left(\boldsymbol{\nu}^{\prime \prime} \cdot \boldsymbol{\kappa}^{\prime}\right) \\
& =\left(\frac{2 k^{2} e}{R c} A\right)^{2}\left(\frac{1}{2 \pi}\right)^{4} \frac{1}{k^{2}} \int_{0}^{k \theta_{a}} d^{2} \kappa d^{2} \kappa^{\prime} \frac{\boldsymbol{\kappa}^{\prime} \cdot \boldsymbol{\kappa}}{\kappa^{\prime 2} \kappa^{2}} e^{i\left(\boldsymbol{\kappa}-\boldsymbol{\kappa}^{\prime}\right) \cdot \boldsymbol{r}} \\
& \times \int \frac{d^{2} r^{\prime}}{r^{\prime}} \frac{d^{2} r^{\prime \prime}}{r^{\prime \prime}} e^{-4 k^{2} \sigma_{\xi}^{2}\left|\boldsymbol{r}^{\prime}-\boldsymbol{r}^{\prime \prime}\right|^{2} / r_{0}^{2}} e^{i \boldsymbol{\kappa}^{\prime} \cdot \boldsymbol{r}^{\prime \prime}-i \boldsymbol{\kappa} \cdot \boldsymbol{r}^{\prime}}\left(\boldsymbol{\nu}^{\prime} \cdot \boldsymbol{\kappa}\right)\left(\boldsymbol{\nu}^{\prime \prime} \cdot \boldsymbol{\kappa}^{\prime}\right) .
\end{aligned}
$$

Note that $\langle I(\boldsymbol{r})\rangle$ involves an 8-dimensional integration!

To calculate the integral (49) we first separate the factor $\left(2 k^{2} e A / R c\right)^{2}$,

$$
\langle I(\boldsymbol{r})\rangle=\left(\frac{2 k^{2} e}{R c} A\right)^{2} J,
$$

where

$$
\begin{aligned}
J & =\left(\frac{1}{2 \pi}\right)^{4} \frac{1}{k^{2}} \int_{0}^{k \theta_{a}} d^{2} \kappa d^{2} \kappa^{\prime} \frac{\boldsymbol{\kappa}^{\prime} \cdot \boldsymbol{\kappa}}{\kappa^{\prime 2} \kappa^{2}} e^{i\left(\boldsymbol{\kappa}-\boldsymbol{\kappa}^{\prime}\right) \cdot \boldsymbol{r}} \\
& \times \int \frac{d^{2} r^{\prime}}{r^{\prime}} \frac{d^{2} r^{\prime \prime}}{r^{\prime \prime}} e^{-4 k^{2} \sigma_{\xi}^{2}\left|\boldsymbol{r}^{\prime}-\boldsymbol{r}^{\prime \prime}\right|^{2} / r_{0}^{2}} e^{i \boldsymbol{\kappa}^{\prime} \cdot \boldsymbol{r}^{\prime \prime}-i \boldsymbol{\kappa} \cdot \boldsymbol{r}^{\prime}}\left(\boldsymbol{\nu}^{\prime} \cdot \boldsymbol{\kappa}\right)\left(\boldsymbol{\nu}^{\prime \prime} \cdot \boldsymbol{\kappa}^{\prime}\right)
\end{aligned}
$$


This integral is somewhat simplified in Appendix A and reduced to a 4dimensional integral (A11) which can be computed numerically. The resulting PSF function, for a particular choice parameters, is illustrated in Fig. (6).

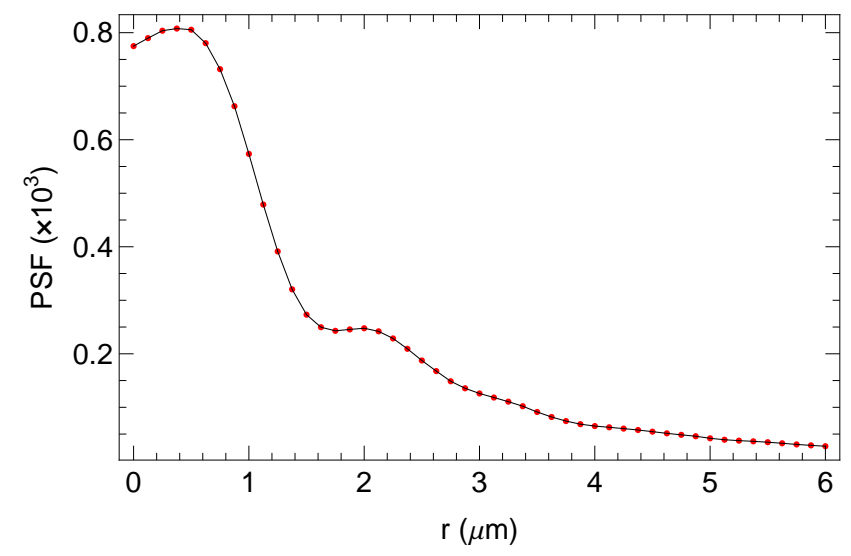

FIG. 6: Plot of the function (A11) for $2 \theta_{r}=0.1, \theta_{a} / 2 \theta_{r}=3$ and $\lambda=0.1 \mu \mathrm{m}$.

\section{COTR IMAGE FROM A ROUGH SURFACE}

We now proceed to calcuation of the COTR image of the beam generated with a rough surface. For this we use (26) averaged over the roughness

$$
\left\langle I_{\mathrm{coh}}\right\rangle=N^{2} \int d^{2} r_{1} d z_{1} d^{2} r_{2} d z_{2} g\left(\boldsymbol{r}_{1}, z_{1}, \boldsymbol{r}_{2}, z_{2}\right)\left\langle\mathfrak{E}_{1}\left(\boldsymbol{r}, \boldsymbol{r}_{1}, z_{1}\right) \cdot \mathfrak{E}_{2}\left(\boldsymbol{r}, \boldsymbol{r}_{2}, z_{2}\right)^{*}\right\rangle .
$$

For the electric field $\mathfrak{E}_{1}$ we use (10) and (42) to which we introduce the phase factor $e^{i k z_{1}}($ see $(21))$

$$
\begin{aligned}
\mathfrak{E}_{1}\left(\boldsymbol{r}, \boldsymbol{r}_{1}, z_{1}\right) & =e^{i k z_{1}} \frac{e^{i k R}}{R} \frac{i}{2 \pi} \frac{k^{2} e}{c \pi \gamma} A \int_{0}^{k \theta_{a}} d^{2} \kappa \frac{\boldsymbol{\kappa}}{\kappa} e^{i \boldsymbol{\kappa} \cdot\left(\boldsymbol{r}-\boldsymbol{r}_{1}\right)} \\
& \times \int d^{2} r^{\prime} K_{1}\left(\frac{k r^{\prime}}{\gamma}\right) e^{-2 i k \xi\left(\boldsymbol{r}^{\prime}\right)} e^{-i \boldsymbol{\kappa} \cdot \boldsymbol{r}^{\prime}} \boldsymbol{\nu}^{\prime} \cdot \frac{\boldsymbol{\kappa}}{\kappa} .
\end{aligned}
$$


We then obtain

$$
\begin{aligned}
\left\langle I_{\text {coh }}\right\rangle & =N^{2}\left(\frac{1}{2 \pi} \frac{k^{2} e}{R c \pi \gamma} A\right)^{2} L \hat{g}(k) \int d^{2} r_{1} d^{2} r_{2} f_{1}\left(\boldsymbol{r}_{1}\right) f_{1}\left(\boldsymbol{r}_{2}\right) \\
& \times \int_{0}^{k \theta_{a}} d^{2} \kappa_{1} \frac{\boldsymbol{\kappa}_{1}}{\kappa_{1}} e^{i \boldsymbol{\kappa}_{1} \cdot\left(\boldsymbol{r}-\boldsymbol{r}_{1}\right)} \int d^{2} r^{\prime} K_{1}\left(\frac{k r^{\prime}}{\gamma}\right) e^{-i \boldsymbol{\kappa}_{1} \cdot \boldsymbol{r}^{\prime}} \boldsymbol{\nu}^{\prime} \cdot \frac{\boldsymbol{\kappa}_{1}}{\kappa_{1}} \\
& \times \int_{0}^{k \theta_{a}} d^{2} \kappa_{2} \frac{\boldsymbol{\kappa}_{2}}{\kappa_{2}} e^{-i \boldsymbol{\kappa}_{2} \cdot\left(\boldsymbol{r}-\boldsymbol{r}_{2}\right)} \int d^{2} r^{\prime \prime} K_{1}\left(\frac{k r^{\prime \prime}}{\gamma}\right) e^{i \boldsymbol{\kappa}_{2} \cdot \boldsymbol{r}^{\prime \prime}} \boldsymbol{\nu}^{\prime \prime} \cdot \frac{\boldsymbol{\kappa}_{2}}{\kappa_{2}} \\
& \times\left\langle e^{-2 i k\left(\xi\left(\boldsymbol{r}^{\prime}\right)-\xi\left(\boldsymbol{(}^{\prime \prime}\right)\right)}\right\rangle .
\end{aligned}
$$

Using again the approximation $K_{1}(k r / \gamma) \approx \gamma / k r$ and (46) and separating the numerical factor (30) we obtain

$$
\left\langle I_{\mathrm{coh}}\right\rangle=|A|^{2} \frac{4 k^{4} e^{2}}{R^{2} c^{2}} N^{2} L J_{\mathrm{coh}}
$$

with

$$
\begin{aligned}
J_{\text {coh }} & =\left(\frac{1}{2 \pi}\right)^{4} k^{-2} \int d^{2} r_{1} d^{2} r_{2} f_{1}\left(\boldsymbol{r}_{1}\right) f_{1}\left(\boldsymbol{r}_{2}\right) \\
& \times \iint \frac{d^{2} r^{\prime}}{r^{\prime}} \frac{d^{2} r^{\prime \prime}}{r^{\prime \prime}} e^{-4 k^{2} \sigma_{\xi}^{2}\left|\boldsymbol{r}^{\prime}-\boldsymbol{r}^{\prime \prime}\right|^{2} / r_{0}^{2}} \\
& \times \iint_{0}^{k \theta_{a}} d^{2} \kappa_{1} d^{2} \kappa_{2}\left(\frac{\boldsymbol{\kappa}_{1}}{\kappa_{1}} \cdot \frac{\boldsymbol{\kappa}_{2}}{\kappa_{2}}\right) e^{i \boldsymbol{\kappa}_{1} \cdot\left(\boldsymbol{r}-\boldsymbol{r}_{1}-\boldsymbol{r}^{\prime}\right)} e^{-i \boldsymbol{\kappa}_{2} \cdot\left(\boldsymbol{r}-\boldsymbol{r}_{2}-\boldsymbol{r}^{\prime \prime}\right)} \\
& \times\left(\boldsymbol{\nu}^{\prime} \cdot \frac{\boldsymbol{\kappa}_{1}}{\kappa_{1}}\right)\left(\boldsymbol{\nu}^{\prime \prime} \cdot \frac{\boldsymbol{\kappa}_{2}}{\kappa_{2}}\right) .
\end{aligned}
$$

Using the Gaussian transverse distribution (35) this integral is simplified and reduced to a one-dimensional integral in Appendix B. For illustration, it is plotted in Fig. 7 and compared with the of COTR from a flat surface.

\section{ACKNOWLEDGMENTS}

I would like to thank H. Loos for useful discussions. 

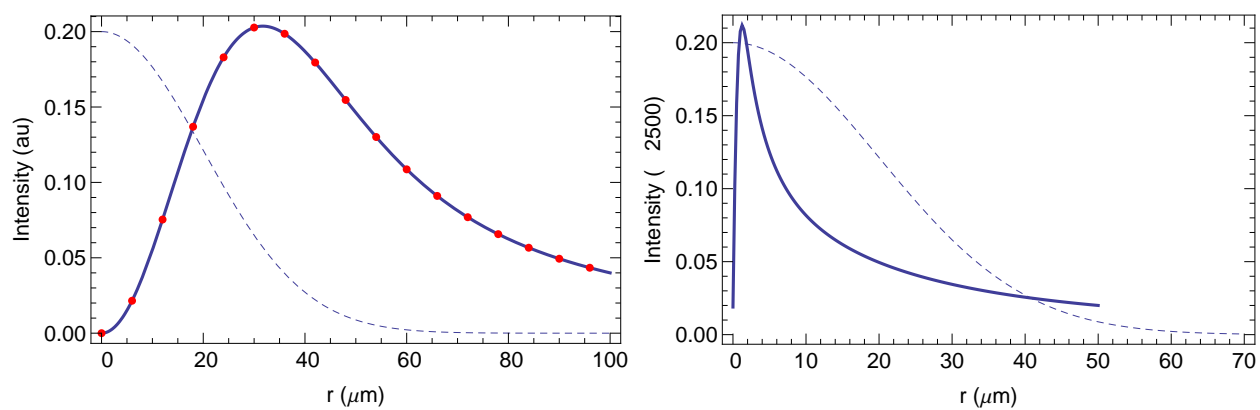

FIG. 7: Comparison of the coherent image from a rough surface according to (38) with the true profile (dashed line). We assumed $2 k \theta_{r} \sigma=20, \theta_{a} \gg \theta_{r}, 2 \theta_{r}=0.1$, $\lambda=0.1 \mu \mathrm{m}$.

This work was supported by the U.S. Department of Energy under Contract No. DE- AC02-76SF00515.

[1] H. Loos, R. Akre, A. Brachmann, F.-J. Decker, Y. Ding, D. Dowell, P. Emma, J. Frisch, S. Gilevich, G. Hays, P. Hering, Z. Huang, R. Iverson, C. LimborgDeprey, A. Miahnahri, S. Molloy, H.-D. Nuhn, J. Turner, J. Welch, W. White, J. Wu, and D. Ratner, in Proceedings of the 2008 FEL Conference, paper THBAU01 (Gyeongju, Korea, 2008).

[2] V. A. Lebedev, Nucl. Inst. and Meth. A 372, 344 (1996).

[3] H. Tanaka, in Proceedings of the 2011 FEL Conference (Shanghai, China, 2011).

[4] J. D. Jackson, Classical Electrodynamics, 3rd ed. (Wiley, New York, 1999).

[5] M. L. Ter-Mikaelian, High-Energy Electromagnetic Processes in Condensed Media (Wiley-Interscience, New York, 1972).

[6] B. M. Bolotovskii and E. A. Galst'yan, Physics-Uspekhi 43, 755 (2000).

[7] S. M. Rytov, Y. A. Kravtsov, and V. I. Tatarskii, Principles of Statistical 
Radiophysics II (Springer, 1988).

\section{APPENDIX A}

We start from performing integration over $\boldsymbol{\kappa}$ and $\boldsymbol{\kappa}^{\prime}$ in (51). Let us denote by $\boldsymbol{R}$ the following vector integral

$$
\boldsymbol{R}=\int_{0}^{k \theta_{a}} d^{2} \kappa \frac{\boldsymbol{\kappa}}{\kappa^{2}} e^{i \boldsymbol{\kappa} \cdot\left(\boldsymbol{r}-\boldsymbol{r}^{\prime}\right)}\left(\boldsymbol{\nu}^{\prime} \cdot \boldsymbol{\kappa}\right)
$$

In tensor notation $K_{\alpha}=J_{\alpha, \beta} \nu_{\beta}^{\prime}$ (summation over repeated indexes is assumed) where

$$
J_{\alpha, \beta}=\int_{0}^{k \theta_{a}} d^{2} \kappa \frac{\kappa_{\alpha} \kappa_{\beta}}{\kappa^{2}} e^{i \kappa \cdot \rho}
$$

with $\boldsymbol{\rho}=\boldsymbol{r}-\boldsymbol{r}^{\prime}$. It is clear that $J_{\alpha, \beta}=C(\rho) \delta_{\alpha, \beta}+D(\rho) \rho_{\alpha} \rho_{\beta}$, where $C$ and $D$ are scalar functions of their arguments. We have $J_{\alpha, \alpha}=3 C+D \rho^{2}$ and $J_{\alpha, \beta} \rho_{\alpha} \rho_{\beta}=C \rho^{2}+D \rho^{4}$, from which we find

$$
C=\frac{1}{2} J_{\alpha, \alpha}-\frac{1}{2 \rho^{2}} J_{\alpha, \beta} \rho_{\alpha} \rho_{\beta}, \quad D=\frac{1}{2 \rho^{2}} J_{\alpha, \alpha}-\frac{3}{2 \rho^{4}} J_{\alpha, \beta} \rho_{\alpha} \rho_{\beta} .
$$

For $J_{\alpha, \alpha}$ and $J_{\alpha, \beta} \rho_{\alpha} \rho_{\beta}$ we obtain

$$
J_{\alpha, \alpha}=\int_{0}^{k \theta_{a}} d^{2} \kappa e^{i \boldsymbol{\kappa} \cdot \boldsymbol{\rho}}=\frac{2 \pi}{\rho} k \theta_{a} J_{1}\left(\rho k \theta_{a}\right),
$$

and

$$
J_{\alpha, \beta} \rho_{\alpha} \rho_{\beta}=\int_{0}^{k \theta_{a}} d^{2} \kappa \frac{(\boldsymbol{\kappa} \cdot \boldsymbol{\rho})^{2}}{\kappa^{2}} e^{i \boldsymbol{\kappa} \cdot \boldsymbol{\rho}}=2 \pi\left(-1+J_{0}\left(k \theta_{a} \rho\right)+k \theta_{a} \rho J_{1}\left(k \theta_{a} \rho\right)\right),
$$

which gives

$$
C(\rho)=\frac{\pi}{\rho^{2}}\left(1-J_{0}(t \rho)\right), \quad D(\rho)=\frac{3 \pi}{\rho^{4}}\left(1-J_{0}(t \rho)-\frac{2}{3} \rho t J_{1}(t \rho)\right),
$$


where $t=k \theta_{a}$. The function $C$ is finite at the origin, but $D$ has a singularity $\sim-\pi t^{2} / 4 \rho^{2}$.

The integral (51) can now be written as

$$
\begin{aligned}
J & =\left(\frac{1}{2 \pi}\right)^{4} \frac{1}{k^{2}} \int \frac{d^{2} r^{\prime}}{r^{\prime}} \frac{d^{2} r^{\prime \prime}}{r^{\prime \prime}} e^{-4 k^{2} \sigma_{\xi}^{2}\left|\boldsymbol{r}^{\prime}-\boldsymbol{r}^{\prime \prime}\right|^{2} / r_{0}^{2}} \\
& \times\left[C\left(\rho^{\prime}\right) \delta_{\alpha, \beta}+D\left(\rho^{\prime}\right) \rho_{\alpha}^{\prime} \rho_{\beta}^{\prime}\right] \nu_{\beta}^{\prime}\left[C\left(\rho^{\prime \prime}\right) \delta_{\alpha, \mu}+D\left(\rho^{\prime \prime}\right) \rho_{\alpha}^{\prime \prime} \rho_{\mu}^{\prime \prime}\right] \nu_{\mu}^{\prime \prime},
\end{aligned}
$$

where $\boldsymbol{\rho}^{\prime}=\boldsymbol{r}-\boldsymbol{r}^{\prime}$ and $\boldsymbol{\rho}^{\prime \prime}=\boldsymbol{r}-\boldsymbol{r}^{\prime \prime}$. Note that even though the function $D$ has a singularity at the origin the integral converges because $D$ is multiplied by $\rho_{\alpha} \rho_{\beta}$. Returning to vector notations, we obtain

$$
\begin{aligned}
J & =\left(\frac{1}{2 \pi}\right)^{4} \frac{1}{k^{2}} \int \frac{d^{2} r^{\prime}}{r^{\prime}} \frac{d^{2} r^{\prime \prime}}{r^{\prime \prime}} e^{-4 k^{2} \sigma_{\xi}^{2}\left|\boldsymbol{r}^{\prime}-\boldsymbol{r}^{\prime \prime}\right|^{2} / r_{0}^{2}} \\
& \times\left[C\left(\rho^{\prime}\right) C\left(\rho^{\prime \prime}\right) \boldsymbol{\nu}^{\prime} \cdot \boldsymbol{\nu}^{\prime \prime}+C\left(\rho^{\prime}\right) D\left(\rho^{\prime \prime}\right)\left(\boldsymbol{\nu}^{\prime} \cdot \boldsymbol{\rho}^{\prime \prime}\right)\left(\boldsymbol{\nu}^{\prime \prime} \cdot \boldsymbol{\rho}^{\prime \prime}\right)\right. \\
& +D\left(\rho^{\prime}\right) C\left(\rho^{\prime \prime}\right)\left(\boldsymbol{\nu}^{\prime} \cdot \boldsymbol{\rho}^{\prime}\right)\left(\boldsymbol{\nu}^{\prime \prime} \cdot \boldsymbol{\rho}^{\prime}\right)+D\left(\rho^{\prime}\right) D\left(\rho^{\prime \prime}\right)\left(\boldsymbol{\rho}^{\prime} \cdot \boldsymbol{\rho}^{\prime \prime}\right)\left(\boldsymbol{\nu}^{\prime} \cdot \boldsymbol{\rho}^{\prime}\right)\left(\boldsymbol{\nu}^{\prime \prime} \cdot \boldsymbol{\rho}^{\prime \prime}\right]
\end{aligned}
$$

It is convenient to change the integration variables from $\boldsymbol{r}^{\prime}$ and $\boldsymbol{r}^{\prime \prime}$ to $\boldsymbol{\rho}^{\prime}$ and $\rho^{\prime \prime}$ respectively,

$$
\begin{aligned}
J & =\left(\frac{1}{2 \pi}\right)^{4} \frac{1}{k^{2}} \int \frac{d^{2} \rho^{\prime}}{r^{\prime}} \frac{d^{2} \rho^{\prime \prime}}{r^{\prime \prime}} e^{-4 k^{2} \sigma_{\xi}^{2}\left|\boldsymbol{\rho}^{\prime}-\boldsymbol{\rho}^{\prime \prime}\right|^{2} / r_{0}^{2}} \\
& \times\left[C\left(\rho^{\prime}\right) C\left(\rho^{\prime \prime}\right) \boldsymbol{\nu}^{\prime} \cdot \boldsymbol{\nu}^{\prime \prime}+C\left(\rho^{\prime}\right) D\left(\rho^{\prime \prime}\right)\left(\boldsymbol{\nu}^{\prime} \cdot \boldsymbol{\rho}^{\prime \prime}\right)\left(\boldsymbol{\nu}^{\prime \prime} \cdot \boldsymbol{\rho}^{\prime \prime}\right)\right. \\
& +D\left(\rho^{\prime}\right) C\left(\rho^{\prime \prime}\right)\left(\boldsymbol{\nu}^{\prime} \cdot \boldsymbol{\rho}^{\prime}\right)\left(\boldsymbol{\nu}^{\prime \prime} \cdot \boldsymbol{\rho}^{\prime}\right)+D\left(\rho^{\prime}\right) D\left(\rho^{\prime \prime}\right)\left(\boldsymbol{\rho}^{\prime} \cdot \boldsymbol{\rho}^{\prime \prime}\right)\left(\boldsymbol{\nu}^{\prime} \cdot \boldsymbol{\rho}^{\prime}\right)\left(\boldsymbol{\nu}^{\prime \prime} \cdot \boldsymbol{\rho}^{\prime \prime}\right]
\end{aligned}
$$

We now introduce the variables $\zeta=2 r k \sigma_{\xi} / r_{0}, \zeta^{\prime}=2 \rho^{\prime} k \sigma_{\xi} / r_{0}$ and $\zeta^{\prime \prime}=$ $2 \rho^{\prime \prime} k \sigma_{\xi} / r_{0}$. We also use the notation $\varkappa=r_{0} / 2 \sigma_{\xi}=1 / 2 \theta_{r}$, and redefine $C$ and $D$ making them dimensionless (and using notations $\tilde{C}$ and $\tilde{D}$ )

$$
\tilde{C}(\zeta)=\frac{1}{\zeta^{2}}\left(1-J_{0}\left(\varkappa \theta_{a} \zeta\right)\right), \quad \tilde{D}(\zeta)=\frac{3}{\zeta^{4}}\left(1-J_{0}\left(\varkappa \theta_{a} \zeta\right)-\frac{2}{3} \varkappa \theta_{a} \zeta J_{1}\left(\varkappa \theta_{a} \zeta\right)\right) .
$$


We then have

$$
\begin{aligned}
J & =\left(\frac{1}{2 \pi}\right)^{4} \frac{\pi^{2}}{\varkappa^{2}} \int \frac{d^{2} \zeta^{\prime}}{\left|\boldsymbol{\zeta}-\boldsymbol{\zeta}^{\prime}\right|} \frac{d^{2} \zeta^{\prime \prime}}{\left|\boldsymbol{\zeta}-\boldsymbol{\zeta}^{\prime \prime}\right|} e^{-\left|\boldsymbol{\zeta}^{\prime}-\boldsymbol{\zeta}^{\prime \prime}\right|^{2}} \\
& \times\left[\tilde{C}\left(\zeta^{\prime}\right) \tilde{C}\left(\zeta^{\prime \prime}\right) \boldsymbol{\nu}^{\prime} \cdot \boldsymbol{\nu}^{\prime \prime}+\tilde{C}\left(\zeta^{\prime}\right) \tilde{D}\left(\zeta^{\prime \prime}\right)\left(\boldsymbol{\nu}^{\prime} \cdot \boldsymbol{\zeta}^{\prime \prime}\right)\left(\boldsymbol{\nu}^{\prime \prime} \cdot \boldsymbol{\zeta}^{\prime \prime}\right)\right. \\
& +\tilde{D}\left(\zeta^{\prime}\right) \tilde{C}\left(\zeta^{\prime \prime}\right)\left(\boldsymbol{\nu}^{\prime} \cdot \boldsymbol{\zeta}^{\prime}\right)\left(\boldsymbol{\nu}^{\prime \prime} \cdot \boldsymbol{\zeta}^{\prime}\right)+\tilde{D}\left(\zeta^{\prime}\right) \tilde{D}\left(\zeta^{\prime \prime}\right)\left(\boldsymbol{\zeta}^{\prime} \cdot \boldsymbol{\zeta}^{\prime \prime}\right)\left(\boldsymbol{\nu}^{\prime} \cdot \boldsymbol{\zeta}^{\prime}\right)\left(\boldsymbol{\nu}^{\prime \prime} \cdot \boldsymbol{\zeta}^{\prime \prime}\right]
\end{aligned}
$$

\section{APPENDIX B}

We first carry out the integration over $r_{1}$ and $r_{2}$ in (56) using

$$
\int d^{2} r_{1} \frac{1}{2 \pi \sigma^{2}} e^{-r_{1}^{2} / 2 \sigma^{2}} e^{-i \boldsymbol{\kappa}_{1} \cdot \boldsymbol{r}_{1}}=e^{-\kappa_{1}^{2} \sigma^{2} / 2}
$$

which gives

$$
\begin{aligned}
J_{\text {coh }} & =\left(\frac{1}{2 \pi}\right)^{4} k^{-2} \iint \frac{d^{2} r^{\prime}}{r^{\prime}} \frac{d^{2} r^{\prime \prime}}{r^{\prime \prime}} e^{-4 k^{2} \sigma_{\xi}^{2}\left|\boldsymbol{r}^{\prime}-\boldsymbol{r}^{\prime \prime}\right|^{2} / r_{0}^{2}} \\
& \times \iint_{0}^{k \theta_{a}} d^{2} \kappa_{1} d^{2} \kappa_{2}\left(\frac{\boldsymbol{\kappa}_{1}}{\kappa_{1}} \cdot \frac{\boldsymbol{\kappa}_{2}}{\kappa_{2}}\right) e^{i \boldsymbol{\kappa}_{1} \cdot\left(\boldsymbol{r}-\boldsymbol{r}^{\prime}\right)-\kappa_{1}^{2} \sigma^{2} / 2} e^{-i \boldsymbol{\kappa}_{2} \cdot\left(\boldsymbol{r}-\boldsymbol{r}^{\prime \prime}\right)-\kappa_{2}^{2} \sigma^{2} / 2} \\
& \times\left(\boldsymbol{\nu}^{\prime} \cdot \frac{\boldsymbol{\kappa}_{1}}{\kappa_{1}}\right)\left(\boldsymbol{\nu}^{\prime \prime} \cdot \frac{\boldsymbol{\kappa}_{2}}{\kappa_{2}}\right) .
\end{aligned}
$$

Assuming a large collection angle in the optical system, $\sigma \gg 1 / k \theta_{a}$, we can extend integration in (B2) over $\kappa_{1}$ and $\kappa_{2}$ to infinity. One can select in (B2) the integral

$$
T=\int_{0}^{\infty} d^{2} \kappa \frac{\boldsymbol{\kappa}}{\kappa^{2}} e^{i \boldsymbol{\kappa} \cdot\left(\boldsymbol{r}-\boldsymbol{r}^{\prime}\right)-\kappa^{2} \sigma^{2} / 2}\left(\boldsymbol{\nu}^{\prime} \cdot \boldsymbol{\kappa}\right),
$$

and perform the integration analogous to (A1). In tensor notation $T_{\alpha}=J_{\alpha, \beta} \nu_{\beta}^{\prime}$ where

$$
J_{\alpha, \beta}=\int_{0}^{\infty} d^{2} \kappa \frac{\kappa_{\alpha} \kappa_{\beta}}{\kappa^{2}} e^{i \kappa \cdot \rho-\kappa^{2} \sigma^{2} / 2},
$$

with $\boldsymbol{\rho}=\boldsymbol{r}-\boldsymbol{r}^{\prime}$. It is clear that $J_{\alpha, \beta}=C(\rho) \delta_{\alpha, \beta}+D(\rho) \rho_{\alpha} \rho_{\beta}$ from which we obtain $J_{\alpha, \alpha}=3 C+D \rho^{2}, J_{\alpha, \beta} \rho_{\alpha} \rho_{\beta}=C \rho^{2}+D \rho^{4}$ and

$$
C=\frac{1}{2} J_{\alpha, \alpha}-\frac{1}{2 \rho^{2}} J_{\alpha, \beta} \rho_{\alpha} \rho_{\beta}, \quad D=\frac{1}{2 \rho^{2}} J_{\alpha, \alpha}-\frac{3}{2 \rho^{4}} J_{\alpha, \beta} \rho_{\alpha} \rho_{\beta}
$$


with

$$
J_{\alpha, \alpha}=\int_{0}^{\infty} d^{2} \kappa e^{i \kappa \cdot \rho-\kappa^{2} \sigma^{2} / 2}=\frac{2 \pi}{\sigma^{2}} e^{-\rho^{2} / 2 \sigma^{2}}
$$

and

$$
J_{\alpha, \beta} \rho_{\alpha} \rho_{\beta}=\int_{0}^{\infty} d^{2} \kappa \frac{(\boldsymbol{\kappa} \cdot \boldsymbol{\rho})^{2}}{\kappa^{2}} e^{i \boldsymbol{\kappa} \cdot \boldsymbol{\rho}-\kappa^{2} \sigma^{2} / 2}=2 \pi\left(e^{-\rho^{2} / 2 \sigma^{2}}\left(\frac{\rho^{2}}{\sigma^{2}}+1\right)-1\right) .
$$

We now find

$$
C(\rho)=\frac{\pi}{\rho^{2}}\left(1-e^{-\rho^{2} / 2 \sigma^{2}}\right), \quad D(\rho)=\frac{\pi}{\rho^{4}}\left(3-3 e^{-\rho^{2} / 2 \sigma^{2}}-\frac{2 \rho^{2}}{\sigma^{2}} e^{-\rho^{2} / 2 \sigma^{2}}\right) .
$$

The function $C$ is finite at the origin, but $D$ has a singularity $\sim 1 / \rho^{2}$ which, however, does not lead to problems because $D$ is multiplied by $\rho_{\alpha} \rho_{\beta}$. We now obtained exactly the result (A8) but with $C$ and $D$ defined by (B8):

$$
\begin{aligned}
J_{\text {coh }} & =\left(\frac{1}{2 \pi}\right)^{4} \frac{1}{k^{2}} \int \frac{d^{2} r^{\prime}}{r^{\prime}} \frac{d^{2} r^{\prime \prime}}{r^{\prime \prime}} e^{-4 k^{2} \sigma_{\xi}^{2}\left|\boldsymbol{r}^{\prime}-\boldsymbol{r}^{\prime \prime}\right|^{2} / r_{0}^{2}} \\
& \times\left[C\left(\rho^{\prime}\right) C\left(\rho^{\prime \prime}\right) \boldsymbol{\nu}^{\prime} \cdot \boldsymbol{\nu}^{\prime \prime}+C\left(\rho^{\prime}\right) D\left(\rho^{\prime \prime}\right)\left(\boldsymbol{\nu}^{\prime} \cdot \boldsymbol{\rho}^{\prime \prime}\right)\left(\boldsymbol{\nu}^{\prime \prime} \cdot \boldsymbol{\rho}^{\prime \prime}\right)\right. \\
& +D\left(\rho^{\prime}\right) C\left(\rho^{\prime \prime}\right)\left(\boldsymbol{\nu}^{\prime} \cdot \boldsymbol{\rho}^{\prime}\right)\left(\boldsymbol{\nu}^{\prime \prime} \cdot \boldsymbol{\rho}^{\prime}\right)+D\left(\rho^{\prime}\right) D\left(\rho^{\prime \prime}\right)\left(\boldsymbol{\rho}^{\prime} \cdot \boldsymbol{\rho}^{\prime \prime}\right)\left(\boldsymbol{\nu}^{\prime} \cdot \boldsymbol{\rho}^{\prime}\right)\left(\boldsymbol{\nu}^{\prime \prime} \cdot \boldsymbol{\rho}^{\prime \prime}\right] .
\end{aligned}
$$

Some further progress can be made if we make an additional assumption. Note that functions $C$ and $D$ vary on the scale of order of $\rho \sim \sigma$ and the factor $e^{-4 k^{2} \sigma_{\xi}^{2}\left|\boldsymbol{r}^{\prime}-\boldsymbol{r}^{\prime \prime}\right|^{2} / r_{0}^{2}}$ effectively limits the difference $\left|\boldsymbol{r}^{\prime}-\boldsymbol{r}^{\prime \prime}\right| \sim r_{0} / k \sigma_{\xi}$. Let us assume that

$$
\sigma \gg \frac{r_{0}}{k \sigma_{\xi}} \sim \frac{\lambda}{\theta_{r}},
$$

that is the transverse size of the beam is larger than the reduces wavelength divided by the roughness angle. In this limit we can set $\rho^{\prime \prime}=\rho^{\prime}$ in the arguments of $C$ and $D$ and carry out integration over $r^{\prime \prime}$ taking into account 
the only dependence of the factor $e^{-4 k^{2} \sigma_{\xi}^{2}\left|\boldsymbol{r}^{\prime}-\boldsymbol{r}^{\prime \prime}\right|^{2} / r_{0}^{2}}$ on $r^{\prime \prime}$. Hence we need to calculate the vector

$$
\mathcal{T}=\int \frac{d^{2} r^{\prime \prime}}{r^{\prime \prime}} e^{-a^{2}\left|\boldsymbol{r}^{\prime}-\boldsymbol{r}^{\prime \prime}\right|^{2}} \boldsymbol{\nu}^{\prime \prime}
$$

where $a=2 k \sigma_{\xi} / r_{0}$. It is clear that it is directed along $\boldsymbol{\nu}^{\prime}, \boldsymbol{T}=\mathcal{T} \boldsymbol{\nu}^{\prime}$, with

$$
\mathcal{T}=\int \frac{d^{2} r^{\prime \prime}}{r^{\prime \prime}} e^{-a^{2}\left|\boldsymbol{r}^{\prime}-\boldsymbol{r}^{\prime \prime}\right|^{2}} \boldsymbol{\nu}^{\prime \prime} \cdot \boldsymbol{\nu}^{\prime}=\frac{2 \pi}{a^{2} r^{\prime}}\left(1-e^{-a^{2} r^{\prime 2}}\right) .
$$

We now substitute this in (B9) and set $\boldsymbol{\rho}^{\prime \prime}=\boldsymbol{\rho}^{\prime}$

$$
\begin{aligned}
J_{\mathrm{coh}} & =\left(\frac{1}{2 \pi}\right)^{3} \frac{1}{k^{2} a^{2}} \int \frac{d^{2} r^{\prime}}{r^{\prime 2}}\left(1-e^{-a^{2} r^{\prime 2}}\right) \\
& \times\left[C\left(\rho^{\prime}\right)^{2}+2 C\left(\rho^{\prime}\right) D\left(\rho^{\prime}\right)\left(\boldsymbol{\nu}^{\prime} \cdot \boldsymbol{\rho}^{\prime}\right)^{2}+D\left(\rho^{\prime}\right)^{2} \rho^{\prime 2}\left(\boldsymbol{\nu}^{\prime} \cdot \boldsymbol{\rho}^{\prime}\right)^{2}\right]
\end{aligned}
$$

Again we use the variables $\zeta=2 r k \sigma_{\xi} / r_{0}, \zeta^{\prime}=2 \rho^{\prime} k \sigma_{\xi} / r_{0}, \zeta^{\prime \prime}=2 \rho^{\prime \prime} k \sigma_{\xi} / r_{0}$, $\varkappa=r_{0} / 2 \sigma_{\xi}=1 / 2 \theta_{r}, \Sigma=2 \sigma k \sigma_{\xi} / r_{0}$ and dimensionless $\tilde{C}$ and $\tilde{D}$

$$
\left.\tilde{C}(\zeta)=\frac{1}{\zeta^{2}}\left(1-e^{-\zeta^{2} / 2 \Sigma^{2}}\right), \quad \tilde{D}(\rho)=\frac{1}{\zeta^{4}}\left(3-3 e^{-\zeta^{2} / 2 \Sigma^{2}}\right)-\frac{2 \zeta^{2}}{\Sigma^{2}} e^{-\zeta^{2} / 2 \Sigma^{2}}\right) .
$$

which gives

$$
\begin{aligned}
J_{\mathrm{coh}} & =\left(\frac{1}{2 \pi}\right)^{3} \frac{\pi^{2}}{\varkappa^{2}} \int \frac{d^{2} \zeta^{\prime}}{\left|\boldsymbol{\zeta}-\boldsymbol{\zeta}^{\prime}\right|^{2}}\left(1-e^{-\left|\boldsymbol{\zeta}-\boldsymbol{\zeta}^{\prime}\right|^{2}}\right) \\
& \times\left[\tilde{C}\left(\zeta^{\prime}\right)^{2}+2 \tilde{C}\left(\zeta^{\prime}\right) \tilde{D}\left(\zeta^{\prime}\right)\left(\boldsymbol{\nu}^{\prime} \cdot \boldsymbol{\zeta}^{\prime}\right)^{2}+\tilde{D}\left(\zeta^{\prime}\right)^{2} \zeta^{\prime 2}\left(\boldsymbol{\nu}^{\prime} \cdot \boldsymbol{\zeta}^{\prime}\right)^{2}\right]
\end{aligned}
$$

